\title{
Atypical Optic Neuritis in an Immunocompetent Patient with Orbital Aspergillosis
}

\author{
Nurul A. M. ${ }^{\mathrm{a}, \mathrm{b}}$, Wan Hazabbah W.H. ${ }^{\mathrm{a}}$, Lakana K.T. ${ }^{\mathrm{b}}$, Maimunah A.M. ${ }^{\mathrm{c}}$ \\ ${ }^{a}$ Department of Ophthalmology and Visual Science, School of Medical Sciences, Health Campus, Universiti Sains Malaysia \\ ${ }^{b}$ Department of Ophthalmology, Hospital Kuala Lumpur \\ 'Department of Ophthalmology, School of Medical Sciences, Universiti Sains Islam Malaysia
}

\begin{abstract}
Keywords
Aspergillosis, immunocompetent, optic

neuropathy, voriconazole

Corresponding Author

Wan Hazabbah Wan Hitam

Department of Ophthalmology and Visual

Science, School of Medical Sciences,

Health Campus, Universiti Sains Malaysia,

16150 Kubang Kerian, Kelantan, Malaysia.

Tel No: +609-7676362

E-mail: hazabbah@usm.my

Received: 24 November 2020; Accepted:

18 February 2021

Doi: https://doi.org/10.31436/imjm.v20i4
\end{abstract}

\begin{abstract}
Aspergillosis infection in immunocompetent individuals is uncommon. Nonspecific presentation could delay diagnosis and management. We describe a case of orbital aspergillosis with cavernous sinus extension in a healthy patient. A $67-$ year-old lady presented with left eye decreased vision, preceded by non-specific headache for three weeks. Visual acuity deteriorated followed by ptosis and left ocular restrictions after corticosteroids therapy. Examination revealed poor left eye visual acuity with ptosis, anisocoria and total ophthalmoplegia. MRI orbit and brain demonstrated a heterogenous left orbital apex lesion extending to cavernous sinus. Transsphenoidal endoscopic biopsy revealed Aspergillus infection at the left retro-orbital space and the sphenoid sinus. Patient was treated with long duration of oral voriconazole. Ptosis and ophthalmoplegia were resolved but left optic atrophy remained. In conclusion, it is important to suspect orbital aspergillosis in cases of subacute painful visual loss. Early identification and prompt treatment may salvage vision in immunocompetent patients.
\end{abstract}

\section{INTRODUCTION}

Aspergillosis is a life-threatening aggressive airborne opportunistic fungal infection in immunocompromised individuals. It is however interesting to note that aspergillosis in immunocompetent hosts also does occur with several cases reported. Aspergillosis may affect various organs as the primary site of infection, or disseminated from a focal point, commonly the lungs or rarely from gastrointestinal tract colonisation or traumatic breached skin lesion. ${ }^{1}$ The aggressive nature of orbital aspergillosis leads to a high mortality and morbidity rate despite the host immunocompetent status. ${ }^{2}$ Early manifestations of other orbital symptoms such as proptosis and ophthalmoplegia no doubt may assist in more prompt diagnosis. Early optic nerve involvement without manifestations of other orbital symptoms is rare.

We present a case of orbital aspergillosis with atypical optic neuritis as the only initial presenting feature in an immunocompetent patient, which was initially treated with corticosteroids. The purpose of this article is to alert clinicians to suspect the possibility of orbital aspergillosis in a case of atypical optic neuritis which was presented sub-acutely. Early identification of the disease may save the patient's sight.

\section{CASE REPORT}

A 67-year-old female with underlying hypertension presented with three weeks history of vague and nonspecific headache. Subsequently she developed gradual blurring of vision in the left eye, associated with pain on ocular movement. On examination, her best corrected visual acuity (BCVA) in the right eye was $6 / 9$ and $6 / 18$ in the left eye, with positive relative afferent pupillary defect (RAPD) in the left eye. Fundoscopy showed normal optic disc appearance in both eyes. Systemic examinations were unremarkable. Infective screening 
was also normal. She was diagnosed as having optic neuritis by the previous ophthalmologist. She was started on intravenous methylprednisolone for three days, followed by oral prednisolone. Her headache slightly improved but her visual acuity deteriorated after two weeks. In view of the progression of her condition, MRI of the brain and orbit was performed and revealed left orbital apex enhancement. She was referred to otorhinolaryngology specialist for further assessment. Transethmoidal orbital endoscopy revealed nonspecific inflammation. A diagnosis of Tolosa-Hunt Syndrome was considered. Patient was started on second course of intravenous methylprednisolone for three days, followed by oral prednisolone. However, her visual acuity declined further and she started to develop ptosis and restricted movement in the left eye. Subsequently, she was referred to our tertiary centre for further management.

On examination, BCVA was 6/9 in the right eye and counting finger (CF) in the left eye. RAPD on the left eye was positive. The patient demonstrated partial ptosis and anisocoria with total ophthalmoplegia in the left eye. Fundoscopy showed normal appearance of optic disc in the right eye and temporal pallor of the optic disc in the left eye. Systemic examination was unremarkable.

Infective screening including blood culture, lumbar puncture analysis, tuberculosis and syphilis screening were normal. CRP and ESR were also normal. Tumour markers were negative. A second MRI scan of the brain and orbit demonstrated a lesion at the left orbital apex extending to the left cavernous sinus with mass effect on the left optic nerve (Figure 1). There were mucosal thickening in the left posterior ethmoidal cells and left sphenoid sinus. Transsphenoidal endoscopic examination by otorhinolaryngology team revealed cheesy lesion surrounded by unhealthy mucosa with necrotic tissue at the left retroorbital space and the sphenoid sinus. There were also surrounding bony erosions. Biopsy specimen was taken and HPE revealed septate hyphae fungal infection suggestive of Aspergillus species (Figure 2).

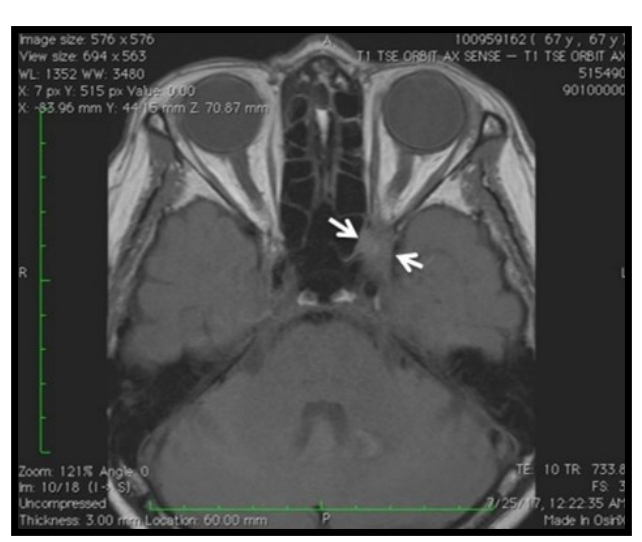

Figure 1: MRI Orbit in T1 sequence on axial plane shows lesion with mixed signal intensity (hyperintense medially and more hypointense laterally) expanding the left orbital apex and extending into the left cavernous sinus (white arrows)

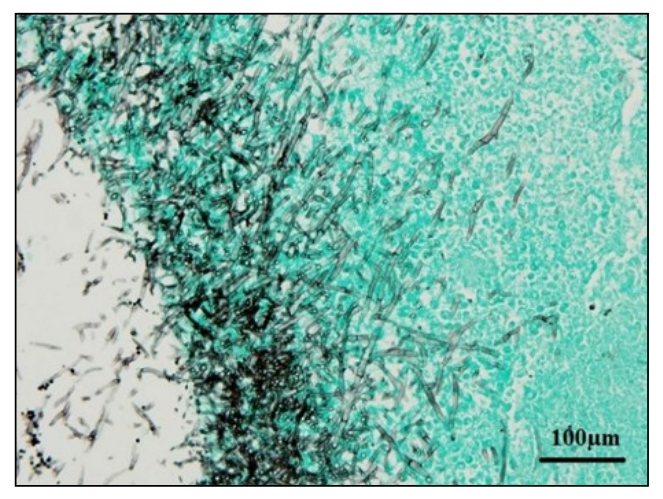

Figure 2: Histopathology slide with Gomori Methanamine Silver stain highlighted the fungal hyphae in black, with characteristic septate branching hyphae suggestive of Aspergillus species (10x40)

The patient was treated with oral voriconazole $200 \mathrm{mg}$ twice daily for a year with close monitoring of liver and renal functions. Repeated MRI after one year showed improvement in the area of the orbital apex and cavernous sinus. Voriconazole was stopped after PET scan showed no active lesion. During the last follow up after six months of cessation of treatment, ptosis and ophthalmoplegia were resolved. Her left eye visual acuity remained poor due to optic atrophy. She is clinically stable with no recurrence of active aspergillosis infection.

\section{DISCUSSION}

Orbital aspergillosis in immunocompetent patient is rare but the problem has been brought to attention by 
Green since 1969.2 Most of the cases are contagious and spread from adjacent sinus in $60 \%-90 \%$ of cases, but some may occur primarily in the orbit. ${ }^{3}$ Invasive type with tissue invasion is the most common with orbital involvement. ${ }^{4}$ This disease may occur at any age as early as 6-months old with a mean age of 34.5 to 47.4 years. $^{5-7}$

Previous largest series described proptosis as the most common presenting feature of orbital aspergillosis in immunocompetent patients, followed by ocular motility restrictions, eyelid swelling, palpable orbital mass and conjunctiva chemosis. ${ }^{5-7}$ Patients may have nasal stuffiness, rhinorrhoea or epistaxis due to concurrent sinus involvement, 4 but none of these symptoms were experienced by our patient. Instead, our patient only demonstrated non-specific headache. As it was subsequently followed by reduced vison and ocular pain, it is not surprising she was treated as atypical optic neuritis and started on high dose corticosteroids. Such presentations naturally add to the diagnostic dilemma. The initial diagnosis in these cases has been reported as bacterial orbital cellulitis, idiopathic orbital inflammatory disease, temporal arteritis and sino-orbital malignancies. , $, 6,9^{2}$

Sinus pathology may alter the normal ventilation process, leading to low oxygen tension environment which promote Aspergillus colonization and spread to the orbit. ${ }^{3}$ It is interesting to note that our patient was asymptomatic for sinusitis despite of the neuroimaging findings which revealed mucosal thickening in the left posterior ethmoidal cell and left sphenoid sinus. Mechanical obstruction of the nasolacrimal duct may also play a role causing superimposed Aspergillus inhabitation after bacterial infection. Hot humid climate, heavy environmental exposure and nutritional deficits have also been linked to the pathogenesis of orbital aspergillosis in immunocompetent patients. 3,8

The insidious nature of this disease is in fact further demonstrated by the fact that the initial MRI showed only left orbital apex enhancement and non-specific inflammatory changes on transethmoidal orbital endoscopy. Non-specific enhancement in neuroimaging should raise a suspicion of orbital aspergillosis, and be thoroughly investigated further. ${ }^{2}$ Involvement of adjacent sinus lining and extension to intracranial structures should be carefully observed. Advanced disease may appear as infiltrative lesion with bony destruction and area of abscesses formation. ${ }^{5-7}$ High dose corticosteroids led to immunosuppression which propagated the Aspergillosis infections leading to worsening of orbital apex syndrome in our patient. Only then was the infection detected via a second MRI and transsphenoidal endoscopic examination. Multiple or repeat biopsies may be necessary to confirm the diagnosis. Typical Aspergillus hyphae are septate with uniform width and dichotomous branching approximately at $45^{\circ}$.

Intracranial complications include cavernous sinus thrombosis, meningitis, brain abscess, cerebral aneurysm, cerebral infarction and subarachnoid haemorrhage. Treatment, naturally, has to be aggressive. Debulking surgery is required in most cases, combined with the use of systemic antifungal therapy. Voriconazole is the drug of choice with a better tolerance and lower toxicity compared to amphotericin B. ${ }^{10}$ Prolonged voriconazole therapy is recommended to ensure the disease is completely treated and to prevent recurrences.

Visual prognosis is poor with a delayed treatment, due to chronic infiltration and compressive insults to the optic nerve. Presentation of subacute painful visual loss warrants early neuro-imaging evaluation including the paranasal sinus assessment. Multiple biopsies may be worthwhile before starting on corticosteroids in such a presentation.

Orbital aspergillosis in immunocompetent individual presents a challenge indeed. Early identification and early treatment of this condition may lead to a better outcome. This case demonstrates that multiple MRI and transsphenoidal endoscopic examination are important especially if the vision deteriorated or does not improve with corticosteroids therapy.

\section{Financial support and sponsorship}

Nil. 


\section{CONFLICTS OF INTEREST}

The authors declare that there is no conflict of interest in the publication of this paper.

\section{REFERENCES}

1. Cheon S, Yang MK, Kim CJ, Kim TS, Song KH, Woo SJ et al. Disseminated aspergillosis in immunocompetent host: a case report and literature review. Mycopathologia 2015; 180:21722.

2. Sivak-Callcott JA, Livesley N, Nugent RA, Rasmussen SL, Saeed P, Rootman J. Localised invasive sino-orbital aspergillosis: characteristics features. Br J Ophthalmol 2004; 88: 681-7.

3. Pushker N, Meel R, Kashyap S, S Bajaj M, Sen S. Invasive aspergillosis of orbit in immunocompetent patients: treatment and outcome. Ophthalmology 2011;118(9):1886-91.

4. Mukherjee B, Raichura ND, Alam MS. Fungal infections of the orbit. Indian J Ophthalmol 2016; 64(5): 337-45.

5. Aggarwal E. Mulay K, Menon V, Sundar G, G. Honavar S, Sharma M. Isolated orbital aspergillosis in immunocompetent patients: a multicenter study. Am J Ophthalmol 2016; 165:125-32.

6. Mody KH, Ali MJ, Vemuganti GK, Nalamada S, Naik MN, Honavar SG. Orbital aspergillosis in immunocompetent patients. Br J Ophthalmol 2014;98(10):1379-84.

7. Adulkar NG, Radhakrishnan S, Vidhya N, Kim U. Invasive sino-orbital fungal infections in immunocompetent patients: a clinico-pathological study. Eye 2019; 33:988-94.

8. Garcia-Giraldo AM, Mora BL, Loaiza-Castano JM, Cedano JA, Rosso F. Invasive fungal infection by Aspergillus flavus in immunocompetent hosts: A case series and literature review. Med Mycol Case Rep 2019;23: 12-5.

9. Mori S, Kurimoto T, Kawara K, Ueda K, Sakamoto M, Keshi $\mathrm{Y}$ et al. The difficulty of diagnosing invasive aspergillosis initially manifesting as optic neuropathy. Case Rep Ophthalmol 2019; 10:11-8.
10. Patterson TF, Thompson GR, Denning DW, Fishman JA, Hadley S, Herbrecht R et al. Practice guidelines for the diagnosis and management of aspergillosis: 2016 update by the infectious diseases society of America. Clin Infect Dis 2016; 63: e1e60. 